\title{
Discrete Isotropies in a Class of Cosmological Models
}

\author{
B.G. SCHMIDT \\ Department of Applied Mathematics and Theoretical Physics, Cambridge
}

Received July 20, 1969

\begin{abstract}
It is shown that a certain class of cosmological models admits discrete isotropies. These models are solutions of Einsteins field equations, characterised by: (1) the matter is described as a perfect fluid, and (2) there exists a group of motions simply transitive on threesurfaces orthogonal to the fluid flow vector.
\end{abstract}

\section{§ 1. Introduction}

In a recent paper [1] Ellis and MacCallum examined in detail properties of solutions of Einstein's field equations for a perfect fluid which admit a three-dimensional group of isometries simply transitive on hypersurfaces orthogonal to the fluid flow. Investigations of the properties of observations in these cosmological models ${ }^{1}$ show that all measurable relations such as the magnitude-red-shift relation in any direction are invariant under certain reflections in the rest space of an observer moving with the matter. The main purpose of this paper is to show the existence of a discrete isotropy group in nearly all of these models, which induces the reflection symmetries mentioned above.

Discrete isometries of a Riemannian space must be treated differently from continuous ones, because no linearised geometrical object such as a killing vector field exists. In $\S 2$ it is shown that if a space admits a transitive group of isometries all further isotropies can be determined and are connected with automorphisms of the Lie algebra.

This is applied in $\S 3$ to positive definite three-spaces with a simply transitive group of isometries and it turns out that all these spaces admit at least one discrete isotropy. The space sections of the cosmological models under consideration therefore admit discrete isotropies.

The question is now whether the isotropy in the space sections is induced by an isotropy group of the space time. The models are determined by solving a Cauchy problem with data given on one of the space sections. $\S 4$ shows that every isometry of the Cauchy data corresponds to an

\footnotetext{
1 A paper on this by the authors of [1] is to appear.
} 
isometry of the development of the data. As a consequence of the constraint equations the discrete isotropies of the space sections leave the extrinsic curvature also invariant. Hence the Cauchy data are invariant under the isotropies and this implies the discrete isotropies of the solution.

\section{§ 2. Discrete Isotropies in Riemannian Spaces}

A Riemannian space admitting a connected simply transitive group of motions can be constructed in the following way. If $G^{n}$ is any $n$-dimensional Lie group then the right translations

$$
R_{a}: x \rightarrow x a \quad x, a \in G^{n}
$$

form a Lie transformation group on $G^{n}$ which is isomorphic to $G^{n}$. To endow $G^{n}$ with a Riemannian metric (of any signature) invariant under all right translations a scalar product $g_{e}(X, Y)$ is defined in the tangent space $T_{e}\left(G^{n}\right)$ of the identity $e$ of $G^{n}$ and then extended on $G^{n}$ by the mappings $R_{a}$. More precisely: for any $a \in G^{n}$ there exists one and only one right translation $R_{a}$ mapping $e$ into $a$. The scalar product in $T_{a}\left(G^{n}\right)$ is therefore uniquely defined by the condition that $\left(R_{a}\right)_{*}: T_{e}\left(G^{n}\right)$ $\rightarrow T_{a}\left(G^{n}\right)$ is an isometry, that is

$$
g_{a}(X, Y):=g_{e}\left(\left(R_{a^{-1}}\right)_{*} X,\left(R_{a-1}\right)_{*} Y\right) .
$$

Now we have to show that this metric is invariant under all right translations. For $R_{b}$ any right translation and $x$ any point of $G^{n}$.

$$
R_{b}=R_{x b} \circ R_{x^{-1}}
$$

holds and therefore we can construct the mapping

by

$$
\left(R_{b}\right)_{*}: T_{x} \rightarrow T_{R_{b}(x)}=T_{x b}
$$

$$
T_{x} \stackrel{\left(R_{x}^{-1}\right)_{e}}{\longrightarrow} T_{e} \stackrel{\left(R_{x b}\right)_{*}}{\longrightarrow} T_{x b} .
$$

Both mappings in (2.5) are isometries. Therefore (2.4) is an isometry and (2.2) is invariant under all right translations. We denote this Riemannian space by $V^{n}=\left(g_{e}, G^{n}\right)$.

It is obvious that the spaces constructed in this way are all spaces with simply transitive groups of isometries.

The Lie algebra of the Killing vector fields is the algebra of the infinitesimal right translations [2]. An important role is played by the infinitesimal left translations, because these are invariant under all right translations and therefore by (2.2) have constant scalar products on $G^{n}$. 
From the construction we see that a space with a simply transitive group of isometries is completely determined by the group $G^{n}$ and the metric at one point. If $G^{n}$ is not simply connected there exists a uniquely determined covering group $\tilde{G}^{n}$. Hence for every space $V^{n}$ with a simply transitive connected group of isometries there exists a simply connected space $\tilde{V}^{n}$, which is locally isometric to $V^{n}$. The local structure is completely determined by the Lie algebra $\underline{G}^{n}$ and a scalar product in $\underline{G}^{n}$. (We can identify $\underline{G}^{n}$ with $T_{e}\left(G^{n}\right)$.)

In general a simply transitive connected group of isometries will not be the maximal group of isometries. We consider here a special way in which further isometries can arise:

Theorem 2.1. In a simply connected space $V^{n}=\left(g_{e}, G^{n}\right)$ any automorphism $\alpha$ of $\underline{G}^{n}$ with $\alpha\left(g_{e}\right)=g_{e}$ induces an isometry of $V^{n}$.

Proof. Since $G^{n}$ is simply connected there is a one-to-one correspondence between automorphisms of $G^{n}$ and $\underline{G}^{n}$, which is given by $\Phi \rightarrow\left(\Phi_{*}\right)_{e}$. For any automorphism $\Phi$ and any $a \in G^{n}$

$$
\Phi=R_{\Phi(a)} \circ \Phi \circ R_{a^{-1}}
$$

holds. That (2.6) is true can be seen by applying both sides to $x \in G^{n}$. If we take the $\Phi$ with $\left(\Phi_{*}\right)_{e}=\alpha$ it can be shown that

$$
\Phi_{*}: T_{a} \rightarrow T_{\Phi(a)}
$$

is an isometry. We can construct this mapping because of (2.6) by

$$
T_{a} \stackrel{\left(R_{a}^{-1}\right)}{\longrightarrow} T_{e} \stackrel{\left(\Phi_{*}\right)}{\longrightarrow} T_{e} \stackrel{\left(R_{a}\right)_{4}}{\longrightarrow} T_{\Phi(a)} .
$$

According to the assumption all mappings in (2.8) are isometries, therefore $\Phi$ is an isometry.

Further isotropies in a space with a simply transitive $G^{n}$ are not necessarily automorphisms of $G^{n}$. But the following is always true:

Theorem 2.2. Suppose $G_{0}^{r}$ is the maximal connected group of isometries acting transitively on $V^{n}$. Then the maximal group of isometries $G^{r}$ is generated by $G_{0}^{r}$ and a discrete group of automorphisms of the Lie algebra $\underline{G}_{0}^{r}$.

Proof. Let us assume that $G_{0}^{r}$ is not maximal. Then there exists an isometry $\Phi$ of $V^{n}$ not contained in $G_{0}^{r}$. If $\Phi$ has no fixed point, there must exist a further isometry $\Psi$ leaving $P$ fixed. $\Psi$ is an automorphism of the Lie algebra of all vector fields on $V^{n}[2]$. As $\Psi$ is an isometry and $G_{0}^{r}$ the maximal connected isometry group on $V^{n}, \Psi$ must map the algebra of killing vector fields of $G_{0}^{r}$ into itself, so that it must be an automorphism of $\underline{G}_{0}^{r}$. 


\section{§3. The Isotropy Group of the Space Sections}

We consider now 3-dimensional, positive definite spaces admitting a simply transitive connected isometry group $G^{3}$. In [1] it is shown that in any of these spaces we can find an orthonormal triad $e_{v}(v=1,2,3)$ with the following commutation relations ([1], (2.16))

$$
\begin{aligned}
& {\left[\boldsymbol{e}_{1}, \boldsymbol{e}_{2}\right]=a \boldsymbol{e}_{2}+n_{3} \boldsymbol{e}_{3},} \\
& {\left[\boldsymbol{e}_{2}, \boldsymbol{e}_{3}\right]=n_{1} \boldsymbol{e}_{1},} \\
& {\left[\boldsymbol{e}_{3}, \boldsymbol{e}_{1}\right]=n_{2} \boldsymbol{e}_{2}-a \boldsymbol{e}_{3},}
\end{aligned}
$$

$a, n_{i}$ are constant, $n_{1} \cdot a=0$. The vector fields $\boldsymbol{e}_{v}$ are invariant under $G^{3}$, that is they are a basis in the Lie algebra of the right invariant vector fields of $G^{3}$. We can choose a basis $\xi_{v}$ of the Killing vector fields with

$$
\left(\xi_{v}\right)_{e}=\left(e_{v}\right)_{e} .
$$

Then the $\boldsymbol{\xi}_{v}$ also have the commutation relations (3.1). To apply Theorem 2.1 of $\S 2$ we must therefore look for orthogonal linear mappings $\alpha$ of $T_{e}$ (in which the $\left(\boldsymbol{e}_{v}\right)_{e}$ are a basis) which are automorphisms of (3.1), that is $\alpha\left[\boldsymbol{e}_{v}, \boldsymbol{e}_{\mu}\right]=\left[\alpha\left(\boldsymbol{e}_{v}\right), \alpha\left(\boldsymbol{e}_{\mu}\right)\right]$. We consider first the groups of class $A$ which are defined by $a=0$. In this case (3.1) reads

$$
\left[\boldsymbol{e}_{1}, \boldsymbol{e}_{2}\right]=n_{3} \boldsymbol{e}_{3} ; \quad\left[\boldsymbol{e}_{2}, \boldsymbol{e}_{3}\right]=n_{1} \boldsymbol{e}_{1} ; \quad\left[\boldsymbol{e}_{3}, \boldsymbol{e}_{1}\right]=n_{2} \boldsymbol{e}_{2} .
$$

It is obvious that the following reflections which we denote by $\mathscr{S}_{v}$ are automorphisms:

$$
\left.\begin{array}{l}
\mathscr{S}_{1}:\left(e_{1}, e_{2}, e_{3}\right) \rightarrow\left(e_{1},-e_{2},-e_{3}\right), \\
\mathscr{S}_{2}:\left(e_{1}, e_{2}, e_{3}\right) \rightarrow\left(-e_{1}, e_{2},-e_{3}\right), \\
\mathscr{S}_{3}:\left(e_{1}, e_{2}, e_{3}\right) \rightarrow\left(-e_{1},-e_{2}, e_{3}\right) .
\end{array}\right\}
$$

A short calculation shows that for $n_{1}=n_{2}$ the rotations in the $\left(\boldsymbol{e}_{1}, \boldsymbol{e}_{2}\right)$ plane are also automorphisms. Similar results hold for $n_{1}=n_{3}$, or $n_{2}=n_{3}$.

We are now able to determine the maximal group $\mathfrak{I}\left(V^{3}\right)$ of motions of these spaces:

$\operatorname{dim} \mathfrak{I}\left(V^{3}\right)=3$ : The maximal isotropy group is discrete, so the isotropies are automorphisms $(\S 2)$. Thus (3.4) is the maximal isotropy group.

$\operatorname{dim} \mathfrak{I}\left(V^{3}\right)=4$ : The space cannot have constant curvature, therefore one of the vectors $\boldsymbol{e}_{v}$ is a distinct Ricci eigenvector. ([1] it is shown that all $\boldsymbol{e}_{v}$ are Ricci eigenvectors for groups of class $A$.)

The isotropy group contains the rotation in the plane orthogonal to the non-degenerated eigenvalue, the isotropies (3.4) and an isotropy which acts in $T_{e}$ as the total reflection if $V^{3}$ is symmetric [3].

$\operatorname{dim} \mathfrak{J}\left(V^{3}\right)=6$ : The spaces are spaces of constant curvature. The isotropy group is the complete 3-dimensional rotation group $\mathcal{O}(3)$. 
Which of the three cases can occur depends on the group type and the values of the $n_{i}$. A list is given in [1], $\S 7$.

The existence of the discrete isotropies (3.4) implies that the vector fields $\boldsymbol{e}_{v}$ are geodesic. $\mathscr{S}_{1}$ corresponds to an isometry leaving the vector field $e_{1}$ invariant. Therefore the first curvature vector $\dot{\boldsymbol{e}}_{1}$ must be invariant. As it lies in the $\boldsymbol{e}_{2}-\boldsymbol{e}_{3}$ plane, $\dot{\boldsymbol{e}}_{1}$ must vanish at $e$ and therefore everywhere. ( $G^{3}$ leaves $\boldsymbol{e}_{v}$ invariant!)

For groups of class $B\left(a \neq 0, n_{1}=0\right)$ the commutation relations are

$$
\left.\begin{array}{l}
{\left[\boldsymbol{e}_{1}, \boldsymbol{e}_{2}\right]=a \boldsymbol{e}_{2}+n_{3} \boldsymbol{e}_{3},} \\
{\left[\boldsymbol{e}_{2}, \boldsymbol{e}_{3}\right]=0,} \\
{\left[\boldsymbol{e}_{3}, \boldsymbol{e}_{1}\right]=n_{2} \boldsymbol{e}_{2}-a \boldsymbol{e}_{3} .}
\end{array}\right\}
$$

In any case we will find that

$$
\mathscr{S}_{1}:\left(e_{1}, e_{2}, e_{3}\right) \rightarrow\left(e_{1},-e_{2},-e_{3}\right)
$$

is an automorphism of (3.5). The only further possible automorphisms are rotations in the $\boldsymbol{e}_{2}-\boldsymbol{e}_{3}$-plane if $n_{2}=n_{3}$, because $\boldsymbol{e}_{1}$ is an invariant vector of $G^{3}[1]$.

For the maximal isometry group $\mathfrak{J}\left(V^{3}\right)$ we find the following: $\operatorname{dim} \mathfrak{I}\left(V^{3}\right)=3: \mathscr{S}_{1}$ is the only isotropy;

$\operatorname{dim} \mathfrak{J}\left(V^{3}\right)=4: \mathscr{S}_{1}$ 1-dim group of rotations, and possible further automorphisms of the 4-dim Lie algebra.

$\operatorname{dim} \mathfrak{J}\left(V^{3}\right)=6: \mathfrak{I}\left(V^{3}\right)=\mathcal{O}(3)$.

\section{$\S 4$. The Isotropy Group of the Cosmological Models}

We consider now the cosmological models of [1]. These are space times $V^{4}$ filled with a perfect fluid which admit a group $G^{3}$ of isometries acting simply transitively on closed spacelike three-surfaces orthogonal to the fluid flow vector. Thus the orthogonal congruence of the group orbits is geodesic. We assume further that every orthogonal geodesic meets every group orbit only once. Under these assumptions the tetrad system defined locally in [1] exists globally on $V^{4}$ and the topology of $V^{4}$ is

$$
V^{4}=G^{3} \times R^{1} .
$$

Using the results of a paper by R. Geroch [4] it is easy to see that every group orbit is a Cauchy hypersurface of $V^{4}$ : Take the orbit $\mathscr{S}:=G^{3} \times\{0\} \subset V^{4}$ then the future Cauchy development $\mathscr{D}^{+}(\mathscr{S})$ (see [9]) of $\mathscr{S}$.consists not merely of points of $\mathscr{S}$ because $\mathscr{S}$ is three-dimensional and spacelike. 
Suppose $\mathscr{D}^{+}(\mathscr{S}) \neq G^{3} \times[0, \infty)$, then $\mathscr{H}^{+}(\mathscr{S}) \neq \theta$, where $\mathscr{H}^{+}(\mathscr{S})$ is the future boundary of $\mathscr{D}^{+}(\mathscr{S})$ (see [9]). $\mathscr{H}^{+}(\mathscr{S})$ must be a group orbit because $\mathscr{D}^{+}(\mathscr{S}), \mathscr{H}^{+}(\mathscr{S})$ are invariant under the group. Therefore $\mathscr{H}^{+}(\mathscr{S})$ is a spacelike three-surface. This is a contradiction, so that $\mathscr{H}^{+}(\mathscr{S})=\theta$. The same holds for $\mathscr{H}^{-}(\mathscr{S})=\theta$ and this implies [4] that $\mathscr{S}$ is a Cauchy surface.

In [1] it is shown that the solutions are determined by a well posed Cauchy initial value problem, which has a unique solution if we give the metric and the extrinsic curvature on $\mathscr{S}$. Therefore we can consider $V^{4}$ as the maximal Cauchy development corresponding to certain initial values, which satisfy the constraint equations.

Now we can prove that every isometry of the Cauchy data corresponds to an isometry of the maximal development.

Theorem 4.1. If $V^{4}$ is the maximal Cauchy development of initial data invariant under a simply transitive $G^{3}$, then every further isometry of the initial data induces an isometry on $V^{4}$.

Proof. Suppose $\tilde{g}, \tilde{d}$ are the Cauchy data on $\mathscr{S}$ invariant under an isometry $\tilde{f}$. We define a diffeomorphism $f: V^{4} \rightarrow V^{4}$ in the following way: If $p \in V^{4}$, there exists a unique geodesic $\mathscr{C}(P)$ through $p$ orthogonal to $\mathscr{S}$. $p^{\prime}=\mathscr{C}(P) \cap S$ is mapped by $\tilde{f}$ into $q^{\prime}$. Then we define $f(P)$ as the point on the orthogonal geodesic $\mathscr{C}^{\prime}$ through $q^{\prime}$ which has the same distance on $\mathscr{C}^{\prime}$ from $q^{\prime}$ as $p$ from $q$ on $\mathscr{C}$. Let $g$ be the solution of the field equations determined by the data $\tilde{g}, \tilde{d}$. Then $f(g)$ is again a solution because the field equations are tensor equations. $g$ and $f(g)$ are determined by the same initial data, therefore by the uniqueness of the solution $f(g)=\mathrm{g}$ must hold so that $f$ is an isometry. Together with the work done in [1] it is now easy to check which of the discrete isotropies determined in $\S 3$ induce discrete isotropies in the considered cosmological models. We have only to look whether the extrinsic curvature is also invariant under the isotropies.

Theorem 4.2. Every model of class $A$ admits at least three discrete isotropies acting in the space sections, given by (3.4).

Proof. The extrinsic curvature is in the notation of [1] given by the expansion tensor $\Theta_{a b}$. Lemma 4.1 in [1] states that as a consequence of the constraints the triad $e_{v}$ is an eigentriad of $\Theta_{a b}$. Therefore $\Theta_{a b}$ is invariant under (3.4). Theorem 4.1 gives then the isotropy in $V^{4}$.

Theorem 4.3. Every model of class $B$ other than the models of group type $V I_{h}$ with $h=-\frac{1}{9}$ admits at least one discrete isotropy of the kind (3.6).

Proof. By [1], Theorem 5.1, $e_{1}$ is an eigenvector of the expansion tensor in all cases besides $V I_{h}\left(h=-\frac{1}{9}\right)$. Therefore $\Theta_{a b}$ is invariant under (3.6). 
Models invariant under a group of type $V I_{h}\left(h=-\frac{1}{9}\right)$ may admit the discrete isotropy (3.6) (if $e_{1}$ is a shear eigendirection) or may not (if $\boldsymbol{e}_{1}$ is not a shear eigendirection). Which of the possible cases occurs is dependent on the choice of the initial data.

\section{§ 5. Isotropy Implies Homogeneity}

The discrete isotropies (3.4) are not only necessary isotropies in a homogeneous model of class $A$ but are also sufficient to imply that a cosmological model is homogeneous.

Theorem 5.1. Suppose $V^{4}$ is a solution of Einsteins field equations for a perfect fluid with the property that $R_{a b c d}, \ldots, R_{a b c d ; e f g}$ at any point are invariant under three linear mappings $\mathscr{S}_{v}$ acting in a spacelike hypersurface (see (3.4)), keeping the orthogonal vector fixed, then $V^{4}$ admits a local group of isometries acting transitively on three-spaces orthogonal to the fluid flow vector.

Proof. The vector fixed under all $\mathscr{S}_{v}$ must be the fluid flow vector $u^{a}$, because it is the unique timelike eigenvector of $R_{a b}$. The congruence $u^{a}$ must be geodesic and hypersurface orthogonal because otherwise $\dot{u}^{a}$, $\omega^{a}$ would not be invariant under all $\mathscr{S}_{v}$. We consider now the intrinsic geometry of one of the three-surface $\mathscr{S}$ orthogonal to $u^{a} . \stackrel{*}{R}_{i k}, \ldots, \stackrel{*}{R}_{i k ; j e m}$ must be invariant under $\mathscr{S}_{v}$. If these tensors determine three vector fields $\boldsymbol{e}_{v}$ covariantly up to the signs, then the rotation coefficients are scalars when we fix an orientation. Therefore their gradients must vanish, otherwise we would have a covariantly determined vector. From this we know that the 3 -spaces $\mathscr{S}$ orthogonal to $u^{a}$ admit a group. With similar arguments it follows that the expansion tensor $\Theta_{a b}$ of the congruence $u^{a}$ must be invariant under the group acting on $\mathscr{S}$. Theorem 4.1 shows now the existence of a local group of isometries on $V^{4}$. When no covariant tetrad is defined by $R_{a b c d}$ and its derivatives up to the third order the space is locally rotationally symmetric. Then the results of [5] show that the space admits a local $G^{4}$ acting on three-spaces.

A very short proof can be given under the assumption that all derivatives of $R_{a b c d}$ are invariant under $\mathscr{S}_{v}$, if one uses the theorem that the number of independent scalars and the dimension of the orbit of the maximal group of isometries add up to the dimension of the space [6]. But as there is so far no general agreement whether this theorem is rigorously proved for indefinite spaces, the longer version above has been given here. 


\section{§ 6. The Topology of the Space Sections}

Every connected Lie group is topologically the direct product of a compact subgroup $K$ and $R^{n}$ [7]. $K$ is uniquely determined up to conjugation. We assume now that the space sections $G^{3}$ are simply connected.

If $G^{3}$ is compact, then $\underline{G}^{3}$ must be semisimple [3]. Then $\underline{G}^{3}$ must be of type $I X$. The group manifold is the covering manifold of the 3dimensional rotation group.

If $G^{3}$ is not compact then we may have (topologically)

$$
G^{3}=K^{1} \times R^{2}, \quad G^{3}=K^{2} \times R^{1}, \quad \text { or } \quad G^{3}=R^{3}
$$

with $K^{1}, K^{2}$ compact. As no semisimple Lie algebra of dimension smaller than three exists the following must hold:

$$
K^{1}=T^{1} \quad \text { or } \quad K^{2}=\left(T^{1} \times T^{1}\right) / N,
$$

[8], where $T^{1}$ is the rotation group in two dimensions and $N$ some discrete subgroup of $T^{1} \times T^{1}$. Because of (6.2) the only simply connected group in (6.1) has the manifold $R^{3}$.

From these spaces we get every space section which is not simply connected by identification of the orbits of an isometry $f$ without fixed points. If $f$ leaves every Killing vector field of $G^{3}$ invariant the new space will also admit a global group, otherwise only a local group.

Acknowledgements. I would like to thank Dr. G. F. R. Ellis and M. A. H. MacCallum for many helpful discussions, and in particular Dr. G. F. R. Ellis for drawing my attention to this problem.

\section{References}

1. Ellis, G. F. R., MacCallum, M. A. H.: Commun. Math. Phys. 12, 108_(1969).

2. Cohn, P.: Lie groups. Cambridge: University Press 1967.

3. Helgason, S.: Differential geometry and symmetric spaces. New York: Academic Press 1962.

4. Geroch, R.: Preprint. Domain of dependence. 1969.

5. Stewart, J. M., Ellis, G. F. R.: J. Math. Phys. 9, 1072 (1968).

6. Jordan, P., Ehlers, J., Kundt, W.: Akad. Wiss. Lit. (Mainz), Abhandl. Math. Nat. Kl. No. 7 (1960).

7. Iwasawa, K.: Ann. Math. 50, 507 (1949).

8. Pontrjagin, L.: Topological groups. Princeton: University Press 1946.

9. Penrose, R.: Structure of space time, Chapter VII of Battelle Rencontres, ed. C. M. DeWitt and J. A. Wheeler. New York: W. A. Benjamin, Inc. 1968.

B. G. Schmidt

I. Institut für Theoretische Physik

der Universität 2 Hamburg 36, Jungiusstr. 9 